\title{
THE REGULARITY OF EUCLIDEAN LIPSCHITZ BOUNDARIES WITH PRESCRIBED MEAN CURVATURE IN THREE-DIMENSIONAL CONTACT SUB-RIEMANNIAN MANIFOLDS
}

\author{
MATTEO GALLI
}

\begin{abstract}
In this paper we consider a set $E \subset \Omega$ with prescribed mean curvature $f \in C(\Omega)$ and Euclidean Lipschitz boundary $\partial E=\Sigma$ inside a three-dimensional contact sub-Riemannian manifold $M$. We prove that if $\Sigma$ is locally a regular intrinsic graph, the characteristic curves are of class $C^{2}$. The result is shape and improves the ones contained in [CCM09] and [GR15b].
\end{abstract}

\section{INTRODUCTION}

The study of the sub-Riemannian area minimizers start with the seminal paper [GN96], where general properties and existence of sets with minimum perimeter are proved in Carnot groups. In the last two decades many effort are been devoted to the develop of an exhaustive theory for minimal surfaces in the sub-Riemannian setting. One of the main difficulties related with this propose is to provide a regularity theory for critical points of the sub-Riemannian area functional.

Nowadays in sub-Riemannian geometry the optimal regularity of critical points of the area is not clear. More precisely, there are only partial results under some assumptions on the regularity of the area-stationary surfaces themselves. Many author focused their attention on the study of minimal $C^{2}$ surfaces or isoperimetric sets with $C^{2}$ boundary, see [DGN07], [DGNP10], [DGNP09], [BSV07], [MSCV08], [RR08], [HRR10], [CHMY05] and [Pau04], among others. A very clear introduction on this first results is presented in the monograph [CDPT07].

The first examples of non-smooth solutions of the Plateau problem with smooth boundary datum in a regular domain are constructed in [Pau06]. Later on, in [CHY07] and [Rit09], the authors find examples of entire graphs over the $x y$-plane, the so called $t$-graphs, that are areaminimizers and Euclidean Lipschitz. On the other hand, we stress that these examples are not $\mathbb{H}$-regular graphs in the sense of [FSS01] and are smooth in their regular part. Another remarkable result is the existence of a non-entire Euclidean Lipschitz $\mathbb{H}$-regular graph that is a global minimizers on a strip, [BSV07, Example 2.8].

In [CHY07] and [CHY09] it is studied the existence and the regularity of $C^{1} t$-graphs in $\mathbb{H}^{n}$ that are critical points of the functional

$$
\mathscr{F}(u)=\int_{\Omega}|\nabla u+\vec{F}|+\int_{\Omega} f u,
$$

on a domain $\Omega \subset \mathbb{R}^{2 n}$, where $\vec{F}$ is a vector field and $f \in L^{\infty}(\Omega)$. When $\vec{F}(x, y)=(-y, x)$, the integral $\int_{\Omega}|\nabla u+\vec{F}|$ is the sub-Riemannian area of the graph over the domain $\Omega$ in the $x y$-plane.

Date: March 11, 2018.

2000 Mathematics Subject Classification. 53C17, 49Q20.

Key words and phrases. Sub-Riemannian geometry, contact manifolds, geometric variational problems, minimal surfaces, prescribed mean curvature surfaces, Heisenberg group.

M. Galli has been supported by the People Programme (Marie Curie Actions) of the European Union's Seventh Framework Programme FP7/2007-2013/ under REA grant agreement n. 607643. 
Among several interesting results, they prove in [CHY09, Thm. A] that, in case $n=1, u \in C^{1}(\Omega)$ is an stationary point of $\mathscr{F}$ and $f \in C^{0}(\Omega)$, the characteristic curves are of class $C^{2}$.

Theorem A in [CHY09] is well-known for $C^{2}$ minimizers and generalizes a previous result by Pauls [Pau06, Lemma 3.3] for $H$-minimal surfaces with components of the horizontal Gauss map in the class $W^{1,1}$. For an explication of what horizontal means we refer to Definition 1.

In [CCM09] the authors consider a Euclidean Lipschitz minimal intrinsic graph of a function $u$ over a domain $\Omega$ inside a vertical plane. Under the assumption that $u$ is a vanishing viscosity minimal graphs (it means that there exists a family $\left\{u_{j}\right\}_{j \in \mathbb{N}}$ of smooth functions $u_{j}: \Omega \rightarrow \mathbb{R}$ converging uniformly to $u$ on compact subsets of $\Omega$ and such that the graph of $u_{j}$ is a Riemannian minimal surface), they prove an intrinsic $C_{\mathbb{H}}^{1, \alpha}$ regularity for the graph of $u$. As a consequence they obtain that the graph of $u$ is foliated by horizontal smooth parabolas.

Very recently, in [GR15b] the authors consider the sub-Riemannian prescribed mean curvature functional

$$
\mathscr{J}(E, B)=P(E, B)+\int_{E \cap B} f,
$$

where $E$ is a set of locally finite sub-Riemannian perimeter in $\Omega, P(E, B)$ is the relative subRiemannian perimeter of $E$ in a bounded open set $B \subset \Omega$, and $f \in L^{\infty}(\Omega)$. If $E \subset \mathbb{H}^{n}$ is the subgraph of a function $t=u(x, y)$ in the Heisenberg group $\mathbb{H}^{n}$, then $\mathscr{J}(E)$ coincides with (1.1) taking $\vec{F}(x, y)=(-y, x)$. In [GR15b], see also [GR15a], they prove that an intrinsic graph of a $C^{1}$ function that is a critical point of the prescribed mean curvature functional is foliated by smooth curves in a 3-dimensional contact manifold. Such curves, usually called characteristic curves, are straight lines in $\mathbb{H}^{1}$, recovering the result first presented in [CHMY05] and [CHY09].

The aim of this paper is to refine the argument in [GR15b] to prove a results in the spirit of [CCM09, BC11] without no assumption on the existence of a sequence of smooth minimal Riemannian graphs approximating the initial sub-Riemannian minimal surface. We shall prove in Theorem 4.2

Let $M$ be a 3-dimensional contact sub-Riemannian manifold, $\Omega \subset M$ a domain, and $E \subset \Omega$ a set of prescribed mean curvature $f \in C(\Omega)$ with Euclidean Lipschitz boundary $\partial E=\Sigma$. If $\Sigma$ is locally a Lipschitz regular graph, then the characteristic curves are of class $C^{2}$.

The regularity of characteristic curves provided in Theorem 4.2 allows us to define in Section 5 a mean curvature function $H$ in the regular part of $\partial E$, that coincides with $f$. As a consequence of the definition of the mean curvature, we shall prove in Proposition 5.3 that characteristic curves are of class $C^{k+2}$ in case $f$ is of class $C^{k}$ when restricted to a characteristic direction. Furthermore we shall characterize the equation of characteristic curves in Proposition 5.5

Let $E \subset \Omega$ be a set of prescribed mean curvature $f \in C(\Omega)$ with Lipschitz boundary $\Sigma$ in a domain $\Omega \subset M$. Then the characteristic curves in $\Sigma-\Sigma_{0}$ are $\nabla$-geodesics of curvature $H$, where $H$ is the mean curvature function and $\nabla$ the pseudo-hermitian connection.

We remark that $\nabla$-geodesics are not in general sub-Riemannian geodesics, see Remark 5.4, and examples of area-stationary surfaces foliated by $\nabla$-geodesics that are not sub-Riemannian geodesics can be found in [Gal14]. We note that Proposition 5.5 provides a characterization of characteristic curves in general 3-dimensional manifolds. This characterization is more natural than the one presented in [CHMY05], [CHY09] and [GR15a]. Indeed the property that a minimal surface is foliated by horizontal straight lines is not preserved by contact changes of 
coordinates. In the Heisenberg structure used in [CCM09] minimal surfaces are foliated by horizontal parabolas, that are $\nabla$-geodesics with vanishing curvature. We also stress that the structure used in [CCM09], see also [BC11], is motivated by application in image reconstruction and vision problems, see for example [CS06], [SCP08] and [Pet08]. Finally it is worth of mention that our results are optimal in view of [BSV07, Example 2.8].

The paper is organized as follows. In Section 2 we provide the necessary background on contact sub-Riemannian manifolds and sets of locally finite perimeter with prescribed mean curvature. In Section 3 we describe Euclidean Lipschitz surfaces that are intrinsic regular graphs. The main result, Theorem 4.2, is proven in Section 4. The consequences on the mean curvature, higher regularity and a geometric characterization for characteristic curves will appear in Section 5 .

\section{Preliminaries}

2.1. Contact sub-Riemannian manifolds. Let $M$ be a 3-dimensional smooth manifold $M$ equipped with a contact form $\omega$ and a sub-Riemannian metric $g_{\mathscr{H}}$ defined on its horizontal distribution $\mathscr{H}:=\operatorname{ker}(\omega)$. By definition, $\left.d \omega\right|_{\mathscr{H}}$ is non-degenerate. We shall refer to $\left(M, \omega, g_{\mathscr{H}}\right)$ as a 3dimensional contact sub-Riemannian manifold. It is well-known that $\omega \wedge d \omega$ is an orientation form in $M$. Since

$$
d \omega(X, Y)=X(\omega(Y))-Y(\omega(X))-\omega([X, Y]),
$$

the horizontal distribution $\mathscr{H}$ is completely non-integrable.

Definition 1. A vector field $X$ is called horizontal if $X \in \mathscr{H}$. A horizontal curve is a $C^{1}$ curve whose tangent vector lies in the horizontal distribution.

The Reeb vector field $T$ in $M$ is the only one satisfying

$$
\omega(T)=1, \quad \mathscr{L}_{T} \omega=0,
$$

where $\mathscr{L}$ is the Lie derivative in $M$.

A canonical contact structure in Euclidean 3-space $\mathbb{R}^{3}$ with coordinates $(x, y, t)$ is given by the contact one-form $\omega_{0}:=d t-y d x$. The associated contact manifold is the Heisenberg group $\mathbb{H}^{1}$. A basis of the horizontal left invariant vector is

$$
X_{0}=\frac{\partial}{\partial x}+y \frac{\partial}{\partial t}, \quad Y_{0}=\frac{\partial}{\partial y},
$$

while the Reeb vector field is

$$
T_{0}=\frac{\partial}{\partial t} .
$$

Darboux's Theorem [Bla02, Thm. 3.1] (see also [GR13]) implies that, given a point $p \in M$, there exists an open neighborhood $U$ of $p$ and a diffeomorphism $\phi_{p}$ from $U$ into an open set of $\mathbb{R}^{3}$ satisfying $\phi_{p}^{*} \omega_{0}=\omega$. Such a local chart will be called a Darboux chart. Composing the map $\phi_{p}$ with a contact transformation of $\mathbb{H}^{1}$ also provides a Darboux chart. This implies we can prescribe the image of a point $p \in U$ and the image of a horizontal direction in $T_{p} M$.

The metric $g_{\mathscr{H}}$ can be uniquely extended to a Riemannian metric $g$ on $M$ by requiring $T$ to be a unit vector orthogonal to $\mathscr{H}$. The Levi-Civita connection associated to $g$ will be denoted by $D$.

The Riemannian volume element in $(M, g)$ will be denoted by $d M$ and coincides with Popp's measure [Mon02, § 10.6], [BR13]. The volume of a set $E \subset M$ with respect to the Riemannian metric $g$ will be denoted by $|E|$. 
2.2. Torsion and the sub-Riemannian connection. The following is taken from [Gal12, § 3.1.2]. In a contact sub-Riemannian manifold, we can decompose the endomorphism $X \in T M \rightarrow D_{X} T$ into its antisymmetric and symmetric parts, which we will denoted by $J$ and $\tau$, respectively,

$$
\begin{aligned}
& 2 g(J(X), Y)=g\left(D_{X} T, Y\right)-g\left(D_{Y} T, X\right), \\
& 2 g(\tau(X), Y)=g\left(D_{X} T, Y\right)+g\left(D_{Y} T, X\right) .
\end{aligned}
$$

Observe that $J(X), \tau(X) \in \mathscr{H}$ for any vector field $X$, and that $J(T)=\tau(T)=0$. Also note that

$$
2 g(J(X), Y)=-g([X, Y], T), \quad X, Y \in \mathscr{H} .
$$

We will call $\tau$ the (contact) sub-Riemannian torsion. We note that our $J$ differs from the one defined in $[\mathrm{Gal13},(2.4)]$ by the constant $g([X, Y], T)$, but plays the same geometric role and can be easily generalized to higher dimensions, [Gal12, § 3.1.2].

Now we define the (contact) sub-Riemannian connection $\nabla$ as the unique metric connection, [Cha06, eq. (I.5.3)], with torsion tensor $\operatorname{Tor}(X, Y)=\nabla_{X} Y-\nabla_{Y} X-[X, Y]$ given by

$$
\operatorname{Tor}(X, Y):=g(X, T) \tau(Y)-g(Y, T) \tau(X)+2 g(J(X), Y) T .
$$

From (2.6) and Koszul formula for the connection $\nabla$ it follows that $\nabla T \equiv 0$.

The standard orientation of $M$ is given by the 3-form $\omega \wedge d \omega$. If $X_{p}$ is horizontal, then the basis $\left\{X_{p}, J\left(X_{p}\right), T_{p}\right\}$ is positively oriented, in fact $(\omega \wedge d \omega)(X, J(X), T)$ and $d \omega(X, J(X))$ have the same sign and

$$
d \omega(X, J(X))=-\omega([X, J(X)])=-g([X, J(X)], T)=g(\operatorname{Tor}(X, J(X)), T)=2 g(J(X), J(X))>0 .
$$

2.3. Perimeter and Lipschitz surfaces. A set $E \subset M$ has locally finite perimeter if, for any bounded open set $B \subset M$, we have

$$
P(E, B):=\sup \left\{\int_{E \cap B} \operatorname{div} U d M: U \text { horizontal, } \operatorname{supp}(U) \subset B,\|U\|_{\infty} \leqslant 1\right\}<+\infty .
$$

The quantity $P(E, B)$ is called the relative perimeter of $E$ in $B$.

Assuming $\partial E$ is a Lipschitz surface and using the Divergence Theorem for Lipschitz boundaries, it is easy to show that the relative perimeter of $E$ in a bounded open set $B \subset M$ coincides with the sub-Riemannian area of $\partial E \cap B$, given by

$$
A(\partial E \cap B)=\int_{\partial E \cap B}\left|N_{h}\right| d(\partial E) .
$$

Here $N$ is the Riemannian unit normal to $\partial E, N_{h}$ is the horizontal projection of $N$ to the horizontal distribution, and $d(\partial E)$ is the Riemannian area measure, all computed with respect the Riemannian metric $g$, see [CDPT07].

2.4. Sets with prescribed mean curvature. Let $\Omega$ a domain in $M$ and let $f: \Omega \rightarrow \mathbb{R}$ a given function. We shall say that a set of locally finite perimeter $E \subset \Omega$ has prescribed mean curvature $f$ on $\Omega$ if, for any bounded open set $B \subset \Omega, E$ is a critical point of the functional

$$
P(E, B)-\int_{E \cap B} f,
$$

where $P(E, B)$ is the relative perimeter of $E$ in $B$, and the integral on $E \cap B$ is computed with respect to the canonical Popp's measure on $M$. The admissible variations for this problem are the flows induced by vector fields with compact support in $B$. 
Let $\Sigma=\partial E$ be a Lipschitz surface in $\Omega$, we say that $\Sigma$ has prescribed mean curvature $f$ if it is a critical point of the functional

$$
A(\Sigma \cap B)-\int_{E \cap B} f
$$

for any bounded open set $B \subset \Omega$.

These definitions are the counterpart of the ones in the Euclidean setting, [Mag12, (12.32) and Remark 17.11]. We note that they are been introduced in the sub-Riemannian setting in [GR15b] .

Remark 2.1. If $E$ is a critical point of the relative perimeter $P(E, B)$ in any bounded open set $B \subset$ $\Omega$, then $E$ has vanishing prescribed mean curvature.

\section{A CHARACTERIZATION OF EUClideAn LipsChitZ REgULAR SURFACES}

The notion of regular surface in the sub-Riemannian geometry has been introduced by Franchi, Serapioni and Serra Cassano, [FSS01, FSSC03]. They defined a regular surface $\Sigma$ as the zero level set of a function $f$, whose horizontal gradient $\nabla^{h}(f)$ never vanishes. The horizontal gradient is defined as

$$
\nabla^{h}(f):=X_{1}(f) X_{1}+X_{2}(f) X_{2},
$$

where $\left\{X_{1}, X_{2}\right\}$ is an orthonormal basis of $\mathscr{H}$. We remark that the function $f$ defining a regular surface $\Sigma$ can be merely $\frac{1}{2}$-Hölder continuous from the Euclidean viewpoint and there exists regular surfaces with fractional Euclidean Hausdorff measure, [KSC04]. This notion can not be applied to Euclidean Lipschitz surfaces since $\nabla^{h}(f)$ is not defined at every point. On other hand it is well-known that a regular surface can be locally described as an intrinsic graph, using an implicit function theorem, see [FSS01, FSSC07, CM06].

Since we are mainly focused on local regularity property, it is not restrictive to describe an Euclidean Lipschitz surface $\Sigma$ as follows. Given a point $p \in \Sigma$, we consider a Darboux chart $\left(U_{p}, \phi_{p}\right)$ such that $\phi_{p}(p)=0$. The metric $g_{\mathscr{H}}$ can be described in this local chart by the matrix of smooth functions

$$
G=\left(\begin{array}{ll}
g_{11} & g_{12} \\
g_{21} & g_{22}
\end{array}\right)=\left(\begin{array}{ll}
g(X, X) & g(X, Y) \\
g(Y, X) & g(Y, Y)
\end{array}\right) .
$$

Under the assumption that $\Sigma$ is an Euclidean Lipschitz surface, we can define $p$ a regular point in $\Sigma$ if there exists (eventually after a Euclidean rotation around the $t$-axis) an open neighborhood $B \cap \Sigma$ that is the graph $G_{u}$ of a Euclidean Lipschitz function $u: D \rightarrow \mathbb{R}$ defined on a domain $D$ in the vertical plane $y=0$. A point $p \in \Sigma$ is singular when it is not regular and we denote by $\Sigma_{0}$ the set of the singular points of $\Sigma$. We remark that an analogous notion was used in 3-dimensional Lie groups in [BC11].

Now we introduce some geometric quantities of the graph $G_{u}$. We have that $G_{u}$ can be parameterized by the map $u: D \rightarrow \mathbb{R}^{3}$ defined by

$$
f_{u}(x, t):=(x, u(x, t), t), \quad(x, t) \in D .
$$

The tangent plane is defined in a.e. point in $G_{u}$ and, when it exists, it is generated by the vectors $E_{1}, E_{2}$ obtained in the following way

$$
\begin{aligned}
& \frac{\partial}{\partial x} \mapsto E_{1}=\left(1, u_{x},-u-x u_{x}\right)=X+u_{x} Y-u T, \\
& \frac{\partial}{\partial t} \mapsto E_{2}=\left(0, u_{t}, 1-x u_{t}\right)=u_{t} Y+T .
\end{aligned}
$$

Then the characteristic direction is given by $Z=\widetilde{Z} /|\widetilde{Z}|$, where

$$
\widetilde{Z}=X+\left(u_{x}+u u_{t}\right) Y \text {. }
$$


We observe that the vector $\widetilde{Z}$ is well-defined and continuous in an Euclidean Lipschitz regular surface since $G_{u}$ is an intrinsic graph, see [ASCV06, BC10].

If $\gamma(s)=(x(s), t(s))$ is a $C^{1}$ curve in $D$, then the lifted curve in $G_{u}$ is

$$
\Gamma(s)=(x(s), u(x(s), t(s)), t(s)-x(s) u(x(s), t(s))) \subset G_{u} .
$$

We stress that even if $\Gamma$ is Lipschitz and the tangent vector

$$
\Gamma^{\prime}(s)=x^{\prime}\left(X+u_{x} Y-u T\right)+t^{\prime}\left(u_{t} Y+T\right)=x^{\prime} X+\left(x^{\prime} u_{x}+t^{\prime} u_{t}\right) Y+\left(t^{\prime}-u x^{\prime}\right) T
$$

exists for a.e. $s$, the vertical component of $\Gamma^{\prime}(s)$ is well defined in all point of $G_{u}$. In particular, horizontal curves in $G_{u}$ satisfy the ordinary differential equation $t^{\prime}=u x^{\prime}$.

Remark 3.1. Since $u \in \operatorname{Lip}(D)$, we have uniqueness of characteristic curves through any given point in $G_{u}$.

Finally, from [GR15b, (4.2)] and the approximation technique in [CMPSC14, Theorem 1.7], we obtain

$$
A\left(G_{u}\right)=\int_{D}\left(g_{22}\left(f_{u}\right)\left(u_{x}+u u_{t}\right)^{2}+2 g_{12}\left(f_{u}\right)\left(u_{x}+u u_{t}\right)+g_{11}\left(f_{u}\right)\right)^{1 / 2} d x d t .
$$

We remark that the functions $g_{i j}$ are bounded on the Darboux chart $U$.

\section{REGULARITY OF PRESCRIBED MEAN CURVATURE SETS WITH LIPSCHITZ BOUNDARY}

First we show a characterization on the area-stationary property of $G_{u}$

Proposition 4.1. Let $G_{u}$ be the graph of an Euclidean Lipschitz function $u: D \rightarrow \mathbb{R}$, defined on a Darboux chart by the parametrization

$$
f_{u}(x, t):=(x, u(x, t), t), \quad(x, t) \in D .
$$

We suppose that the area of $G_{u}$ is defined by (3.1) and $G_{u}$ has prescribed mean curvature $f$. Then

$$
\int_{D}\left(K v+M\left(v_{x}+u v_{t}+v u_{t}\right) d x d t=0\right.
$$

for any $v \in C_{0}^{1}(D)$, where the functions $K, K_{1}$ and $M$ are given by

and

$$
\begin{gathered}
K=K_{1}-f \operatorname{det}(G), \\
K_{1}=\frac{\frac{\partial g_{22}}{\partial y} \cdot\left(u_{x}+u u_{t}\right)^{2}+2 \frac{\partial g_{12}}{\partial y} \cdot\left(u_{x}+u u_{t}\right)+\frac{\partial g_{11}}{\partial y}}{2\left(g_{22} \cdot\left(u_{x}+u u_{t}\right)^{2}+2 g_{12}\left(u_{x}+u u_{t}\right)+g_{11}\right)^{1 / 2}},
\end{gathered}
$$

Proof. First we prove a first variation formula for $A\left(G_{u}\right)$ for variations of $G_{u}$ given by $G_{u+s v}$, where $v \in C_{0}^{1}(D)$ and $s$ is a small parameter. Denoting $w=u+s v$, we have

$$
\left.\frac{d}{d s}\right|_{s=0} A\left(G_{w}\right)=\left.\int_{D} \frac{d}{d s}\right|_{s=0}\left(g_{22}\left(f_{w}\right)\left(w_{x}+w w_{t}\right)^{2}+2 g_{12}\left(f_{w}\right)\left(w_{x}+w w_{t}\right)+g_{11}\left(f_{w}\right)\right)^{1 / 2} d x d t .
$$

Taking into account that

$$
\frac{d}{d s} g_{i j}\left(f_{w}\right)=\frac{\partial}{\partial y} g_{i j}\left(f_{w}\right) \cdot \frac{d}{d s} w=\frac{\partial}{\partial y} g_{i j}\left(f_{w}\right) \cdot v
$$


and

we conclude

$$
\frac{d}{d s}\left(w_{x}+w w_{t}\right)=v_{x}+u v_{t}+v u_{t}
$$

$$
\left.\frac{d}{d s}\right|_{s=0} A\left(G_{w}\right)=\int_{D}\left(K_{1} v+M\left(v_{x}+u v_{t}+v u_{t}\right) d x d t=0 .\right.
$$

Here $K$ and $M$ are defined in the statement of the proposition. On the other hand it is easy to see that

which conclude the proof.

$$
\left.\frac{d}{d s}\right|_{s=0} \int_{\text {subgraph } G_{u+s v}} f=\int_{D} f \operatorname{det}(G) v d x d t,
$$

Now we are able to prove the main regularity result

Theorem 4.2. Let $M$ be a 3-dimensional contact sub-Riemannian manifold, $\Omega \subset M$ a domain, and $E \subset \Omega$ a set of prescribed mean curvature $f \in C(\Omega)$ with Euclidean Lipschitz boundary $\partial E=\Sigma$. If $\Sigma$ is locally a Lipschitz regular graph, then the characteristic curves are of class $C^{2}$.

Proof. Given any point $p \in \Sigma-\Sigma_{0}$, it is sufficient show the result in the Darboux chart $(U, \phi)$ introduced in Section 3. We consider

$$
Z=\frac{X+\left(u_{x}+u u_{t}\right) Y}{\left(g_{22}\left(u_{x}+u u_{t}\right)^{2}+2 g_{12}\left(u_{x}+u u_{t}\right)+g_{11}\right)^{1 / 2}},
$$

the tangent vector to an horizontal curve in $\mathbb{G}_{u}$. The function $M$ introduced in (4.2) coincides with $g(Z, Y) \circ f_{u}$. Then

$$
1=|Z|^{2}=\operatorname{det}(G)^{-1}\left(g_{22} g(Z, X)^{2}-2 g_{12} g(Z, X) g(Z, Y)+g_{11} g(Z, Y)^{2}\right)
$$

and

$$
g(Z, X)=\frac{g_{12} g(Z, Y) \pm\left(\operatorname{det}(G)\left(g_{22}-g(Z, Y)^{2}\right)\right)^{1 / 2}}{g_{22}} .
$$

By Schwarz's inequality $g(Z, Y)^{2} \leqslant g(Y, Y)=g_{22}$. Inequality is strict since otherwise $Y$ and $Z$ would be collinear. Hence $g(Z, X)$ has the same regularity as $g(Z, Y)$ by (4.3).

Now we proceed as in the proof of [GR15b, Theorem 4.1], see also [GR15a, Theorem 3.5]. Assume the point $p \in G_{u}$ corresponds to the point $(a, b)$ in the $x t$-plane. The curve $s \mapsto(s, t(s))$ is (a reparameterization of the projection of) a characteristic curve if and only if the function $t(s)$ satisfies the ordinary differential equation $t^{\prime}(s)=u(s, t(s))$. For $\varepsilon$ small enough, we consider the solution $t_{\varepsilon}$ of equation $t_{\varepsilon}^{\prime}(s)=u\left(s, t_{\varepsilon}(s)\right)$ with initial condition $t_{\varepsilon}(a)=b+\varepsilon$, and define $\gamma_{\varepsilon}(s):=\left(s, t_{\varepsilon}(s)\right)$, with $\gamma=\gamma_{0}$. We may assume that, for small enough $\varepsilon$, the functions $t_{\varepsilon}$ are defined in the interval $[a-r, a+r]$ for some $r>0$.

We consider the parameterization

$$
F(\xi, \varepsilon):=\left(\xi, t_{\varepsilon}(\xi)\right)=(s, t)
$$

near the characteristic curve through $(a, b)$. The jacobian of this parameterization is given by

$$
\operatorname{det}\left(\begin{array}{cc}
1 & t_{\varepsilon}^{\prime} \\
0 & \partial t_{\varepsilon} / \partial \varepsilon
\end{array}\right)=\frac{\partial t_{\varepsilon}}{\partial \varepsilon}
$$

which is positive because of the choice of initial condition for $t_{\varepsilon}$ and the fact that the curves $\gamma_{\varepsilon}(s)$ foliate a neighborhood of $(a, b)$. We remark that $t_{\varepsilon}$ is a Lipschitz function with respect to $\varepsilon$, [Tes12, Theorem 2.9], and the jacobian of the parametrization is well-defined. Using the implicit function theorem for Lipschitz functions, [Cla76, Pap05], any function $\varphi$ can be considered as a 
function of the variables $(\xi, \varepsilon)$ by making $\tilde{\varphi}(\xi, \varepsilon):=\varphi\left(\xi, t_{\varepsilon}(\xi)\right)$. Changing variables, and assuming the support of $\varphi$ is contained in a sufficiently small neighborhood of $(a, b)$, we can express the integral (4.1) as

$$
\int_{I}\left\{\int_{a-r}^{a+r}\left(K \tilde{\varphi}+M\left(\frac{\partial \tilde{\varphi}}{\partial \xi}+2 \tilde{\varphi} \tilde{u}_{t}\right)\right) \frac{\partial t_{\varepsilon}}{\partial \varepsilon} d \xi\right\} d \varepsilon
$$

where $I$ is a small interval containing 0 . Instead of $\tilde{\varphi}$, we can consider the function $\tilde{\zeta}=$ $\tilde{\varphi} h /\left(t_{\varepsilon+h}-t_{\varepsilon}\right)$, where $h$ is a sufficiently small real parameter. We get that

tends a.e. to

$$
\frac{\partial \tilde{\zeta}}{\partial \xi}=\frac{\partial \tilde{\varphi}}{\partial \xi} \cdot \frac{h}{t_{\varepsilon+h}-t_{\varepsilon}}-2 \tilde{\varphi} \cdot \frac{\tilde{u}(\xi, \varepsilon+h)-\tilde{u}(\xi, \varepsilon)}{t_{\varepsilon+h}-t_{\varepsilon}} \cdot \frac{h}{t_{\varepsilon+h}-t_{\varepsilon}}
$$

$$
\frac{\partial \tilde{\varphi} / \partial \xi}{\partial t_{\varepsilon} / \partial \varepsilon}-\frac{2 \tilde{\varphi} \tilde{u}_{t}}{\partial t_{\varepsilon} / \partial \varepsilon}
$$

when $h \rightarrow 0$. So using that $G_{u}$ is area-stationary we have that

$$
\int_{I}\left\{\int_{a-r}^{a+r} \frac{h}{t_{\varepsilon+h}-t_{\varepsilon}}\left(K \tilde{\varphi}+M\left(\frac{\partial \tilde{\varphi}}{\partial \xi} \cdot+2 \tilde{\varphi} \cdot\left(\tilde{u}_{t}-\frac{\tilde{u}(\xi, \varepsilon+h)-\tilde{u}(\xi, \varepsilon)}{t_{\varepsilon+h}-t_{\varepsilon}}\right)\right)\right) \frac{\partial t_{\varepsilon}}{\partial \varepsilon} d \xi\right\} d \varepsilon
$$

vanishes. Furthermore, letting $h \rightarrow 0$ we conclude

$$
\int_{I}\left\{\int_{a-r}^{a+r}\left(K \tilde{\varphi}+M \frac{\partial \tilde{\varphi}}{\partial \xi}\right) d \xi\right\} d \varepsilon=0
$$

Let now $\eta: \mathbb{R} \rightarrow \mathbb{R}$ be a positive function with compact support in the interval $I$ and consider the family $\eta_{\rho}(x):=\rho^{-1} \eta(x / \rho)$. Inserting a test function of the form $\eta_{\rho}(\varepsilon) \psi(\xi)$, where $\psi$ is a $C^{\infty}$ function with compact support in $(a-r, a+r)$, making $\rho \rightarrow 0$, and using that $G_{u}$ is area-stationary we obtain

$$
\int_{a-r}^{a+r}\left(K(0, \xi) \psi(\xi)+M(0, \xi) \psi^{\prime}(\xi)\right) d \xi=0
$$

for any $\psi \in C_{0}^{\infty}((a-r, a+r))$. By [GR15b, Lemma 4.2], the function $M(0, \xi)$, which is the restriction of $g(Z, Y)$ to the characteristic curve, is a $C^{1}$ function on the curve. By equation (4.3), the restriction of $g(Z, X)$ to the characteristic curve is also $C^{1}$. This proves that horizontal curves are of class $C^{2}$.

\section{THE MEAN CURVATURE AND A CHARACTERIZATION OF CHARACTERISTIC CURVES}

In this section we introduce the definition of the mean curvature of a regular Lipschitz boundary and present some consequences. This results partially follows [GR15b, § 4] and we will omit some proofs.

Given a Lipschitz surface $\Sigma \subset M$ such that the characteristic curves are $C^{2}$-smooth in $\Sigma \backslash \Sigma_{0}$, we define the mean curvature of $\Sigma$ at $p \in \Sigma \backslash \Sigma_{0}$ by

$$
H(p):=g\left(\nabla_{Z} Z, v_{h}\right)(p) .
$$

This is the standard definition of mean curvature for Euclidean $C^{2}$ surfaces, see e.g. [CHMY05, (2.1)], [RR08, (4.8)] and [DGN07, (1.4)]. Using the regularity Theorem 4.2 we get

Proposition 5.1. Let $\Omega \subset M$ be a domain and $E \subset \Omega$ a set of prescribed mean curvature $f \in C(\Omega)$ with Lipschitz boundary $\Sigma$ with $H \in L_{\text {loc }}^{1}(\Sigma)$. Then the first variation of the functional (2.9) induced by an horizontal vector field $U \in C_{0}^{1}(\Omega)$ is given by

$$
\int_{\Sigma} H\left|N_{H}\right| g\left(U, v_{h}\right) d \Sigma-\int_{\Sigma} f\left|N_{H}\right| g\left(U, v_{h}\right) d \Sigma .
$$


Proof. We first observe that formula

$$
\left.\frac{d}{d s}\right|_{s=0} A\left(\Sigma_{s}\right)=-\int_{\Sigma} H\left|N_{H}\right| g\left(U, v_{h}\right) d \Sigma
$$

can be proved as in [Gal13, § 6] with a standard approximation argument. Note that even $N$ is defined a.e. on $\Sigma,\left|N_{H}\right|$ and $v_{h}$ are well defined on $\Sigma-\Sigma_{0}$. On the other hand, it is well-known that

$$
\left.\frac{d}{d s}\right|_{s=0}\left(\int_{\phi_{s}(\Omega)} f\right)=-\int_{\Sigma} f\left|N_{H}\right| g\left(U, v_{h}\right) d \Sigma
$$

see e.g. [Mag12, 17.8].

The following corollary is an immediate consequence of (5.2)

Corollary 5.2. Let $E \subset \Omega$ be a set of prescribed mean curvature $f \in C(\Omega)$ with Lipschitz boundary $\Sigma$ in a domain $\Omega \subset M$. Assume $H \in L_{\text {loc }}^{1}(\Sigma)$. Then $H(p)=f(p)$ for any $p \in \Sigma \backslash \Sigma_{0}$.

Finally we can improve the regularity of Theorem 4.2 assuming an higher regularity for $f$.

Proposition 5.3. Let $E \subset \Omega$ be a set of prescribed mean curvature $f \in C(\Omega)$ with $C^{1}$ boundary $\Sigma$ in a domain $\Omega \subset M$. Assume that $f$ is also $C^{k}$ in the $Z$-direction, $k \geqslant 1$. Then the characteristic curves of $\Sigma$ are of class $C^{k+2}$ in $\Sigma \backslash \Sigma_{0}$.

The proof of last proposition is the analogous of [GR15b, Proposition 5.3]. A remarkable consequence of Proposition 5.3 is that critical points of perimeter (eventually under a volume constraint) with Lipschitz boundaries are foliated by smooth horizontal curves on its regular set.

To provide a geometric characterization of characteristic curves we need the following definition

Definition 2. A curve $\gamma$ is a $\nabla$-geodesic of curvature $h: I \rightarrow \mathbb{R}$ if

$$
\nabla_{\dot{\gamma}} \dot{\gamma}+h J(\dot{\gamma})=0
$$

Remark 5.4. We stress that a $\nabla$-geodesic of curvature $h$ is a sub-Riemannian geodesic if and only if it satisfies

$$
\dot{\gamma}(h)=g(\tau(\dot{\gamma}), \dot{\gamma})
$$

see [Rum94, Proposition 15].

Proposition 5.5. Let $E \subset \Omega$ be a set of prescribed mean curvature $f \in C(\Omega)$ with Lipschitz boundary $\Sigma$ in a domain $\Omega \subset M$. Then the characteristic curves in $\Sigma-\Sigma_{0}$ are $\nabla$-geodesic of curvature $H$, where $H$ is defined in (5.1).

Proof. Let $Z$ the tangent unit vector to a characteristic curve $\gamma: I \rightarrow \Sigma-\Sigma_{0}$. Then we have

$$
\begin{aligned}
\nabla_{Z} Z & =g\left(\nabla_{Z} Z, Z\right)+g\left(\nabla_{Z} Z, T\right)+g\left(\nabla_{Z} Z, v_{h}\right) \\
& =g\left(\nabla_{Z} Z, v_{h}\right) \\
& =-H J(Z) .
\end{aligned}
$$

Remark 5.6. The result in Proposition 5.5 was shown for $C^{2}$ CMC surfaces in $\mathbb{H}^{1}$, [DGNP10, DGN07, RR08], in Sasakian space forms, [Ros12], and in general 3-dimensional contact manifolds, [Gal13, HP08, HP10, Gal14]. 


\section{REFERENCES}

[ASCV06] Luigi Ambrosio, Francesco Serra Cassano, and Davide Vittone. Intrinsic regular hypersurfaces in Heisenberg groups. J. Geom. Anal., 16(2):187-232, 2006.

[BC10] Francesco Bigolin and Francesco Serra Cassano. Distributional solutions of Burgers' equation and intrinsic regular graphs in Heisenberg groups. J. Math. Anal. Appl., 366(2):561-568, 2010.

[BC11] D. Barbieri and G. Citti. Regularity of minimal intrinsic graphs in 3-dimensional sub-Riemannian structures of step 2. J. Math. Pures Appl. (9), 96(3):279-306, 2011.

[Bla02] David E. Blair. Riemannian geometry of contact and symplectic manifolds, volume 203 of Progress in Mathematics. Birkhäuser Boston, Inc., Boston, MA, 2002.

[BR13] Davide Barilari and Luca Rizzi. A formula for Popp's volume in sub-Riemannian geometry. Anal. Geom. Metr. Spaces, 1:42-57, 2013.

[BSV07] Vittorio Barone Adesi, Francesco Serra Cassano, and Davide Vittone. The Bernstein problem for intrinsic graphs in Heisenberg groups and calibrations. Calc. Var. Partial Differential Equations, 30(1):17-49, 2007.

[CCM09] Luca Capogna, Giovanna Citti, and Maria Manfredini. Regularity of non-characteristic minimal graphs in the Heisenberg group $\mathbb{H}^{1}$. Indiana Univ. Math. J., 58(5):2115-2160, 2009.

[CDPT07] Luca Capogna, Donatella Danielli, Scott D. Pauls, and Jeremy T. Tyson. An introduction to the Heisenberg group and the sub-Riemannian isoperimetric problem, volume 259 of Progress in Mathematics. Birkhäuser Verlag, Basel, 2007.

[Cha06] Isaac Chavel. Riemannian geometry, volume 98 of Cambridge Studies in Advanced Mathematics. Cambridge University Press, Cambridge, second edition, 2006. A modern introduction.

[CHMY05] Jih-Hsin Cheng, Jenn-Fang Hwang, Andrea Malchiodi, and Paul Yang. Minimal surfaces in pseudohermitian geometry. Ann. Sc. Norm. Super. Pisa Cl. Sci. (5), 4(1):129-177, 2005.

[CHY07] Jih-Hsin Cheng, Jenn-Fang Hwang, and Paul Yang. Existence and uniqueness for $p$-area minimizers in the Heisenberg group. Math. Ann., 337(2):253-293, 2007.

[CHY09] Jih-Hsin Cheng, Jenn-Fang Hwang, and Paul Yang. Regularity of $C^{1}$ smooth surfaces with prescribed $p$-mean curvature in the Heisenberg group. Math. Ann., 344(1):1-35, 2009.

[Cla76] F. H. Clarke. On the inverse function theorem. Pacific J. Math., 64(1):97-102, 1976.

[CM06] G. Citti and M. Manfredini. Implicit function theorem in Carnot-Carathéodory spaces. Commun. Contemp. Math., 8(5):657-680, 2006.

[CMPSC14] Giovanna Citti, Maria Manfredini, Andrea Pinamonti, and Francesco Serra Cassano. Smooth approximation for intrinsic Lipschitz functions in the Heisenberg group. Calc. Var. Partial Differential Equations, 49(34):1279-1308, 2014.

[CS06] G. Citti and A. Sarti. A cortical based model of perceptual completion in the roto-translation space. J. Math. Imaging Vision, 24(3):307-326, 2006.

[DGN07] D. Danielli, N. Garofalo, and D. M. Nhieu. Sub-Riemannian calculus on hypersurfaces in Carnot groups. Adv. Math., 215(1):292-378, 2007.

[DGNP09] D. Danielli, N. Garofalo, D. M. Nhieu, and S. D. Pauls. Instability of graphical strips and a positive answer to the Bernstein problem in the Heisenberg group $\mathbb{H}^{1}$. J. Differential Geom., 81(2):251-295, 2009.

[DGNP10] Donatella Danielli, Nicola Garofalo, Duy-Minh Nhieu, and Scott D. Pauls. The Bernstein problem for embedded surfaces in the Heisenberg group $\mathbb{H}^{1}$. Indiana Univ. Math. J., 59(2):563-594, 2010.

[FSS01] Bruno Franchi, Raul Serapioni, and Francesco Serra Cassano. Rectifiability and perimeter in the Heisenberg group. Math. Ann., 321(3):479-531, 2001.

[FSSC03] Bruno Franchi, Raul Serapioni, and Francesco Serra Cassano. On the structure of finite perimeter sets in step 2 Carnot groups. J. Geom. Anal., 13(3):421-466, 2003.

[FSSC07] Bruno Franchi, Raul Serapioni, and Francesco Serra Cassano. Regular submanifolds, graphs and area formula in Heisenberg groups. Adv. Math., 211(1):152-203, 2007.

[Gal12] Matteo Galli. Area-stationary surfaces in contact sub-Riemannian manifolds. PhD thesis, Universidad de Granada, Available at http://hera.ugr.es/tesisugr/21013020.pdf, 2012.

[Gal13] Matteo Galli. First and second variation formulae for the sub-Riemannian area in three-dimensional pseudoHermitian manifolds. Calc. Var. Partial Differential Equations, 47(1-2):117-157, 2013.

[Gal14] Matteo Galli. On the classification of complete area-stationary and stable surfaces in the subriemannian Sol manifold. Pacific J. Math., 271(1):143-157, 2014.

[GN96] Nicola Garofalo and Duy-Minh Nhieu. Isoperimetric and Sobolev inequalities for Carnot-Carathéodory spaces and the existence of minimal surfaces. Comm. Pure Appl. Math., 49(10):1081-1144, 1996.

[GR13] Matteo Galli and Manuel Ritoré. Existence of isoperimetric regions in contact sub-Riemannian manifolds. $J$. Math. Anal. Appl., 397(2):697-714, 2013. 
[GR15a] Matteo Galli and Manuel Ritoré. Area-stationary and stable surfaces of class $C^{1}$ in the sub-Riemannian Heisenberg group $\mathbb{H}^{1}$. Adv. Math., 285:737-765, 2015.

[GR15b] Matteo Galli and Manuel Ritoré. Regularity of $C^{1}$ surfaces with prescribed mean curvature in threedimensional contact sub-Riemannian manifolds. Calc. Var. Partial Differential Equations, 54(3):2503-2516, 2015.

[HP08] Robert K. Hladky and Scott D. Pauls. Constant mean curvature surfaces in sub-Riemannian geometry. $J$. Differential Geom., 79(1):111-139, 2008.

[HP10] Robert K. Hladky and Scott D. Pauls. Minimal surfaces in the roto-translation group with applications to a neuro-biological image completion model. J. Math. Imaging Vision, 36(1):1-27, 2010.

[HRR10] Ana Hurtado, Manuel Ritoré, and César Rosales. The classification of complete stable area-stationary surfaces in the Heisenberg group $\mathbb{H}^{1}$. Adv. Math., 224(2):561-600, 2010.

[KSC04] Bernd Kirchheim and Francesco Serra Cassano. Rectifiability and parameterization of intrinsic regular surfaces in the Heisenberg group. Ann. Sc. Norm. Super. Pisa Cl. Sci. (5), 3(4):871-896, 2004.

[Mag12] Francesco Maggi. Sets of finite perimeter and geometric variational problems, volume 135 of Cambridge Studies in Advanced Mathematics. Cambridge University Press, Cambridge, 2012. An introduction to geometric measure theory.

[Mon02] Richard Montgomery. A tour of subriemannian geometries, their geodesics and applications, volume 91 of Mathematical Surveys and Monographs. American Mathematical Society, Providence, RI, 2002.

[MSCV08] Roberto Monti, Francesco Serra Cassano, and Davide Vittone. A negative answer to the Bernstein problem for intrinsic graphs in the Heisenberg group. Boll. Unione Mat. Ital. (9), 1(3):709-727, 2008.

[Pap05] Marco Papi. On the domain of the implicit function and applications. J. Inequal. Appl., (3):221-234, 2005.

[Pau04] Scott D. Pauls. Minimal surfaces in the Heisenberg group. Geom. Dedicata, 104:201-231, 2004.

[Pau06] Scott D. Pauls. H-minimal graphs of low regularity in $\mathbb{H}^{1}$. Comment. Math. Helv., 81(2):337-381, 2006.

[Pet08] Jean Petitot. Neurogéométrie de la vision. Éditions de l'École Polytechnique, Palaiseau, 2008. Modèles mathématiques et physiques des architectures fonctionnelles. [Mathematical and physical models of the functional architectures].

[Rit09] Manuel Ritoré. Examples of area-minimizing surfaces in the sub-Riemannian Heisenberg group $\mathbb{H}^{1}$ with low regularity. Calc. Var. Partial Differential Equations, 34(2):179-192, 2009.

[Ros12] César Rosales. Complete stable CMC surfaces with empty singular set in Sasakian sub-Riemannian 3manifolds. Calc. Var. Partial Differential Equations, 43(3-4):311-345, 2012.

[RR08] Manuel Ritoré and César Rosales. Area-stationary surfaces in the Heisenberg group $\mathbb{H}^{1}$. Adv. Math., 219(2):633-671, 2008.

[Rum94] Michel Rumin. Formes différentielles sur les variétés de contact. J. Differential Geom., 39(2):281-330, 1994.

[SCP08] Alessandro Sarti, Giovanna Citti, and Jean Petitot. The symplectic structure of the primary visual cortex. Biol. Cybernet., 98(1):33-48, 2008.

[Tes12] Gerald Teschl. Ordinary differential equations and dynamical systems, volume 140 of Graduate Studies in Mathematics. American Mathematical Society, Providence, RI, 2012.

Dipartimento di Matematica, Università di Bologna, Piazza di Porta S. Donato 5, 40126 Bologna, Italy

E-mail address: matteo.galli8@unibo.it 